\title{
Classificação dos pacientes segundo o risco de complicações e mortalidade após cirurgias cardíacas eletivas
}

\author{
Carina Aparecida Marosti Dessotte ${ }^{1}$, Mariana Lopes Figueiredo ${ }^{2}$, Hélen Francine Rodrigues ${ }^{3}$, \\ Rejane Kiyomi Furuya ${ }^{4}$, Lidia Aparecida Rossi ${ }^{5}$, Rosana Aparecida Spadoti Dantas ${ }^{6}$
}

\footnotetext{
${ }^{1}$ Enfermeira, Doutora em Enfermagem. Professora Doutora da Escola de Enfermagem de Ribeirão Preto da Universidade de São Paulo (EERP/USP). Ribeirão Preto, SP, Brasil. E-mail: camarosti@usp.br.

${ }^{2}$ Enfermeira. Ribeirão Preto, SP, Brasil. Email: marialf09@hotmail.com.

${ }^{3}$ Enfermeira. Discente do Programa de Pós-Graduação em Enfermagem Fundamental, nível Mestrado, da EERP/USP. Ribeirão Preto, SP, Brasil. Email: hfrodrigues.usp@gmail.com.

${ }^{4}$ Enfermeira, Doutora em Enfermagem. Ribeirão Preto, SP, Brasil. E-mail: re.furuya@gmail.com.

${ }^{5}$ Enfermeira, Doutora em Enfermagem Fundamental. Professora Titular da EERP/USP. Ribeirão Preto, SP, Brasil. Email: rizzardo@eerp.usp.br.

${ }^{6}$ Enfermeira, Doutora em Enfermagem. Professora Associada da EERP/USP. Ribeirão Preto, SP, Brasil. E-mail: rsdantas@eerp.usp.br.
}

Recebido: 23/09/2015.

Aceito: 30/11/2015.

Publicado: 31/03/2016.

Como citar esse artigo:

Dessotte CAM, Figueiredo ML, Rodrigues HF, Furuya RK, Rossi LA, Dantas RAS.

Classificação dos pacientes segundo o risco de complicações e mortalidade após cirurgias cardíacas eletivas. Rev. Eletr. Enf. [Internet]. 2016 [acesso em: ___ ];18:e1140. Disponível em: http://dx.doi.org/10.5216/ree.v18.37736.

\section{RESUMO}

O objetivo da investigação foi classificar os pacientes segundo o risco de complicações e mortalidade após cirurgias cardíacas eletivas, utilizando o Sistema de Escore Clínico de Risco de Tuman. Estudo descritivo, desenvolvido em um hospital universitário do interior de São Paulo, Brasil, entre agosto de 2013 e fevereiro de 2015. Uma amostra consecutiva e não probabilística foi constituída por pacientes submetidos à primeira cirurgia de revascularização do miocárdio e/ou cirurgias para correção de valvulopatias, e com agendamento eletivo de suas cirurgias. Resultados: participaram 125 pacientes. A maioria apresentou baixo risco para o desenvolvimento de complicações pósoperatórias e mortalidade ( $n=110 ; 88 \%)$. É esperada uma taxa de complicação pós-operatória de $14,6 \%$ e uma taxa de 3,3\% de mortalidade para esses pacientes. Nessa pesquisa, a maioria dos pacientes submetidos pela primeira vez às cirurgias cardíacas eletivas apresentou baixo risco para o desenvolvimento de complicações pósoperatórias e mortalidade.

Descritores: Cirurgia Torácica; Mortalidade; Enfermagem Perioperatória.

\section{INTRODUÇÃO}

As doenças cardiovasculares (DCV) são as principais causas de morbimortalidade tanto em países desenvolvidos como em desenvolvimento ${ }^{(1)}$. 
Atualmente, com o avanço da ciência na área da saúde, muitos pacientes podem se beneficiar de abordagens minimamente invasivas para o tratamento das DCV. Entretanto, a cirurgia cardíaca ainda pode ser o único tratamento indicado e disponível para um grande número de pacientes.

De acordo com os dados disponíveis no Departamento de Informática do Sistema Único de Saúde (DATASUS), nos últimos cinco anos foram realizadas 179.135 cirurgias cardíacas de revascularização do miocárdio (RVM) e/ou correção de valvopatias, no Brasil. Desse total, 64\% ( $n=114.599$ ) foram RVM, 30\% ( $n=54.129)$ foram correções de valvopatias e 6,0\% ( $n=10.407)$ foram cirurgias combinadas de RVM e correção de valvopatias. O número total de óbitos nesse período foi de 12.827 , acometendo $50 \%$ ( $n=6.421)$ dos pacientes submetidos à RVM, 37\% $(n=4.790)$ dos pacientes submetidos à correção de valvopatias e $13 \%$ $(n=1.616)$ dos pacientes submetidos às cirurgias combinadas de RVM e correção de valvopatias ${ }^{(2)}$.

Em decorrência do advento das abordagens minimamente invasivas, recentemente, houve uma mudança significativa no perfil dos pacientes submetidos à cirurgia cardíaca. A cirurgia de RVM, por exemplo, está sendo indicada mais tardiamente para pacientes com lesões mais grave, com maior número de comorbidades pré-operatórias e em pacientes mais idosos ${ }^{(3)}$. Por sua vez, as cirurgias para correções de valvopatias também estão sendo indicadas para pacientes mais idosos, com grau mais avançado de insuficiência e/ou estenose valvar e portadores de doenças crônicas associadas ${ }^{(4)}$.

Frente ao atual perfil dos pacientes submetidos à cirurgia cardíaca, inúmeros trabalhos têm abordado a importância da avaliação clínica pré-operatória, com o objetivo de predizer o risco de complicações pósoperatórias e/ou de mortalidade ${ }^{(3,5-10)}$.

Para a realização dessa avaliação clínica, a equipe multidisciplinar pode utilizar escores de classificação de risco de complicações pós-operatórias e mortalidade de pacientes submetidos à cirurgia cardíaca, disponíveis e validados na literatura. Tais escores são utilizados desde o final da década de 80, porém, até o momento, ainda não há consenso sobre o melhor a ser utilizado. Mesmo com a falta desse consenso, não há dúvidas sobre a importância dessa avaliação pré-operatória, que poderá subsidiar a decisão terapêutica, o planejamento da assistência de enfermagem, além de permitir a adoção de estratégias individualizadas para a prevenção de complicações e mortalidade. Além disso, a avaliação e o acompanhamento dos escores de risco dos pacientes, com suas respectivas taxas de complicações pós-operatórias e mortalidade, são considerados excelentes indicadores de qualidade dos serviços hospitalares ${ }^{(10)}$.

$\mathrm{Na}$ literatura, encontram-se estudos que correlacionaram o perfil clínico pré-operatório do paciente submetido à cirurgia cardíaca com possíveis complicações pós-operatórias e/ou mortalidade ${ }^{(3,5-10)}$, mas a utilização de escores validados para essa avaliação é escassa ${ }^{(7,10)}$.

A classificação pré-operatória sistematizada dos pacientes, segundo o risco de complicações e mortalidade após cirurgias cardíacas, através de escores validados na literatura, poderá trazer subsídios para o planejamento da assistência multidisciplinar a esses pacientes. Não há investigações publicadas dessa natureza na Instituição em que foi desenvolvido o presente estudo, tampouco outros realizados na área de abrangência do Departamento Regional de Saúde de Ribeirão Preto - DRS XIII. Consideramos importante 
essa avaliação, uma vez que esse DRS é composto por 26 municípios, e atende uma população de aproximadamente 1.433 .463 habitantes ${ }^{(11)}$.

Assim, esta pesquisa teve como objetivo classificar os pacientes segundo o risco de complicações e mortalidade após cirurgias cardíacas eletivas de RVM, correção de valvulopatias e RVM combinada com correção de valvopatias, atendidos em um hospital universitário do interior paulista.

\section{MÉTOdO}

Trata-se de um estudo descritivo, desenvolvido em um hospital universitário do interior de São Paulo, Brasil, pertencente ao Departamento Regional de Saúde de Ribeirão Preto - DRS XIII.

Os dados foram coletados entre agosto de 2013 e fevereiro de 2015. Uma amostra consecutiva e não probabilística foi constituída por sujeitos de ambos os sexos, com idade acima de 18 anos, submetidos à primeira cirurgia de RVM e/ou cirurgias para correção de valvulopatias, e com agendamento eletivo de suas cirurgias.

Foram excluídos os pacientes que não apresentaram condições cognitivas para responder o questionário, avaliadas pelo "Mini Exame do Estado Mental - MEEM" (12), na versão adaptada para o português ${ }^{(13)}$; que apresentaram descompensação clínica da doença cardíaca, no dia que antecedeu a cirurgia (presença de dispneia, precordialgia e entubação orotraqueal); e que tiveram o agendamento eletivo das cirurgias com menos de 12 horas de antecedência.

O projeto de pesquisa foi elaborado de acordo com os preceitos éticos da Resolução do Conselho Nacional de Saúde n. 466 de dezembro de 2012, e foi aprovado pelo Comitê de Ética em Pesquisa da Escola de Enfermagem de Ribeirão Preto-USP, com o número CAAE: 27214414.9.0000.5393. Cada participante do estudo foi devidamente esclarecido quanto à pesquisa. Foi realizada a leitura do Termo de Consentimento Livre e Esclarecido, o qual foi assinado pelo participante e pelo pesquisador, após o consentimento em participar da pesquisa.

Os dados de caracterização sociodemográfica e clínica, bem como os dados utilizados para a classificação de risco de complicações pós-operatórias e mortalidade, foram coletados no dia que antecedeu a cirurgia cardíaca, por meio de entrevistas individuais com os participantes e consulta aos prontuários.

Para a caracterização dos participantes, foi elaborado um instrumento de coleta de dados. Foi realizada a validação de face e conteúdo desse instrumento por um comitê de quatro juízes, com vasta experiência na área de cardiologia e cirurgia cardiovascular. Para cada um dos juízes foi solicitado para avaliar todos os itens do instrumento quanto à pertinência (se os itens expressavam verdadeira relação com a proposta do estudo) e clareza (se os itens estavam descritos de forma compreensível). Caso o juiz avaliasse um item como não claro ou não pertinente, havia espaço para sugestões, bem como espaço para o acréscimo de novos itens.

O instrumento constou de variáveis sociodemográficas (data de nascimento; sexo; escolaridade em anos completos; estado civil; desempenho de atividades remuneradas e renda mensal familiar) e clínicas 
(data de internação; comorbidades; diagnóstico principal e cirurgia realizada). A idade dos participantes foi calculada subtraindo a data de nascimento da data da entrevista. Para o cálculo da carga tabágica, utilizamos o número de cigarros consumidos por dia relatado pelos pacientes, dividido por 20 e multiplicado pelo número de anos em que o paciente fumou ${ }^{(14)}$.

As variáveis "data de nascimento; sexo (feminino ou masculino); data de internação; presença de comorbidades (peso e altura para o cálculo do índice de massa corpórea; dislipidemia; hipotireoidismo; insuficiência renal crônica; insuficiência cardíaca; diabetes mellitus; hipertensão arterial sistêmica e fibrilação atrial); diagnóstico principal (doença arterial coronariana e sua manifestação clínica e/ou tipo de valvopatia e válvula acometida) e cirurgia realizada (RVM, troca de válvula, plastia de válvula, RVM + troca de válvula, RVM + plastia de válvula)" foram coletadas do prontuário médico do paciente. Já as variáveis "tabagismo atual ou pregresso; escolaridade em anos completos; estado civil; desempenho de atividades remuneradas e renda mensal familiar" foram obtidas através de entrevistas individuais com os participantes.

Para a classificação dos pacientes segundo o risco de complicações e mortalidade após cirurgias cardíacas, utilizamos o "Sistema de Escore Clínico de Risco de Tuman”"(15), na sua versão validada para o português ${ }^{(10)}$. No Quadro 1 estão apresentadas as variáveis que compõem o Sistema de Escore Clínico de Risco de Tuman.

Quadro 1: Sistema de escore clínico de risco, segundo Tuman e colaboradores ${ }^{(10)}$.

\begin{tabular}{|l|c|}
\hline \multicolumn{1}{|c|}{ Fator pré-operatório } & Escore \\
\hline Cirurgia de emergência & 4 \\
\hline Idade & \\
\hline 65-74 anos & 1 \\
\hline$\geq 75$ anos & 2 \\
\hline Disfunção renal & 2 \\
\hline Infarto do miocárdio prévio & \\
\hline 3-6 meses & 1 \\
\hline <3 meses & 2 \\
\hline Sexo feminino & 2 \\
\hline Cirurgia cardíaca prévia & 2 \\
\hline Hipertensão pulmonar & 2 \\
\hline Doença cerebrovascular & 2 \\
\hline Troca de válvula múltipla ou RVM + troca valvar & 2 \\
\hline Troca de válvula aórtica ou mitral & 1 \\
\hline Insuficiência cardíaca congestiva & \\
\hline Disfunção do ventrículo esquerdo & \\
\hline
\end{tabular}

A variável "disfunção renal” (creatinina pré-operatória maior que 1,4 mg/dl) ${ }^{(15)}$ foi coletada do exame de sangue realizado no dia que antecedeu a cirurgia. Já as variáveis "hipertensão pulmonar" (pressão da artéria pulmonar 25 milímetros de mercúrio acima da pressão arterial sistêmica) e "disfunção do ventrículo esquerdo" (fração de ejeção do ventrículo esquerdo< $35 \%$ ) $^{(15)}$ foram coletadas do exame mais recente de ecocardiograma, realizado no pré-operatório. 
Para a classificação dos pacientes, utilizamos os seguintes pontos de corte ${ }^{(15)}$ : pacientes com escore de 0 a 5 - baixo risco (taxa de mortalidade esperada é de 3,3\% e taxa de complicações pós-operatórias (PO) de 14,6\%); escore de 6 a 9 - risco moderado, (taxa de mortalidade esperada de 10,0\% e de complicações PO de $34,4 \%$ ); e escore igual ou maior que 10 - alto risco (taxa de mortalidade esperada é de $25,4 \%$ e de complicações PO de 61,0\%).

Todos os dados foram analisados usando programa estatístico. Foram realizadas análises descritivas de frequência simples para variáveis nominais ou categóricas, de tendência central (média e mediana) e dispersão (desvio-padrão, valores mínimo e máximo) para as variáveis contínuas.

\section{RESULTADOS}

No período de coleta de dados, de agosto de 2013 a fevereiro de 2015, foram realizadas 204 cirurgias cardíacas no referido hospital. Desse total, 48 pacientes não atenderam aos critérios de inclusão (28 pacientes devido ao tipo de cirurgia; 18 reoperações e dois pacientes menores de 18 anos), 24 pacientes foram excluídos (15 tiveram o agendamento com menos de 12 horas de antecedência, sete não apresentavam condições clínicas para responder os questionários e dois não apresentavam condições cognitivas) e sete se recusaram a participar do estudo. A amostra final foi composta por 125 pacientes.

A caracterização sociodemográfica dos participantes encontra-se na Tabela 1.

Tabela 1: Caracterização sociodemográfica da amostra. Ribeirão Preto, SP, Brasil, agosto 2013 - fevereiro 2015.

\begin{tabular}{|c|c|c|c|c|c|}
\hline \multirow{2}{*}{ Variável } & \multicolumn{5}{|c|}{ Amostra total $(n=125)$} \\
\hline & $\mathrm{n}(\%)$ & Média & Desvio Padrão & Mínimo & Máximo \\
\hline \multicolumn{6}{|l|}{ Sexo } \\
\hline Masculino & $82(65,6)$ & & & & \\
\hline Idade & & 59,10 & 11,8 & 25,8 & 87,7 \\
\hline \multicolumn{6}{|l|}{ Estado civil } \\
\hline Casado/União consensual & $92(73,6)$ & & & & \\
\hline \multicolumn{6}{|c|}{ Desempenho de atividade remunerada } \\
\hline Inativo & $83(66,4)$ & & & & \\
\hline Escolaridade em anos & & 5,2 & 4,2 & zero & 20 \\
\hline Renda mensal familiar (em reais) & & 2.340 & 2.438 & 70 & 20.000 \\
\hline Número de dependentes da renda & & 2,2 & 9,3 & 01 & 15 \\
\hline
\end{tabular}

A caracterização clínica da amostra, segundo o diagnóstico principal, encontra-se na Tabela 2. 
Tabela 2: Caracterização clínica da amostra, segundo o diagnóstico principal. Ribeirão Preto, SP, Brasil, agosto 2013 - fevereiro 2015.

\begin{tabular}{lc}
\hline \multicolumn{1}{c}{ Variável } & Amostra Total $\mathbf{n}(\%)$ \\
\hline Diagnóstico principal (n=125) & $61(48,8)$ \\
Doença Arterial Coronariana (DAC) & $53(42,4)$ \\
Valvopatia & $11(8,8)$ \\
DAC+ valvopatia & $33(45,8)$ \\
Manifestação clínica da DAC (n=72) & $17(23,6)$ \\
Angina estável & $13(18,1)$ \\
IAM recente & $09(12,5)$ \\
IAM prévio & \\
Angina instável & $29(45,3)$ \\
Válvula acometida (n=64) & $21(32,8)$ \\
Aórtica & $13(20,3)$ \\
Mitral & $01(1,6)$ \\
Aórtica + mitral & \\
Aórtica + mitral + tricúspide & $33(51,6)$ \\
Tipo de valvopatia ( $\mathbf{n}=\mathbf{6 4 )}$ & $22(34,4)$ \\
Estenose + insuficiência & $09(14,1)$ \\
Insuficiência &
\end{tabular}

$\mathrm{IAM}^{\mathrm{a}}=$ Infarto Agudo do Miocárdio.

Quanto à presença de comorbidades dos pacientes no pré-operatório, encontramos que a maioria dos pacientes apresentava hipertensão arterial sistêmica $(n=88 ; 70,4 \%)$, sobrepeso/obesidade $(n=74 ; 59,2 \%)$ e dislipidemia ( $n=64 ; 51,2 \%)$. Encontramos ainda 49 pacientes $(39,2 \%)$ com Diabetes mellitus; $22(17,6 \%)$ com insuficiência cardíaca; 14 (11,2\%) com hipotireoidismo; 11 (8,8\%) com insuficiência renal crônica e 10 (8,0\%) pacientes com fibrilação atrial crônica.

Vinte e dois pacientes $(17,6 \%)$ relataram tabagismo atual e $51(40,8 \%)$ tabagismo pregresso. Os pacientes que relataram tabagismo atual (fumantes até 30 dias antes da internação para a realização da cirurgia) fumaram em média 22,3 (D.P.=21,0) cigarros por dia. 0 tempo médio de tabagismo foi de 35,4 anos (D.P.=15,0). Sendo assim, esses pacientes apresentaram em média 43,7 (D.P. $=45,5$ ) anos-maço de tabagismo. Já os pacientes que relataram tabagismo pregresso fumaram em média 19,4 (D.P. $=15,0$ ) cigarros por dia. 0 tempo médio de tabagismo pregresso foi de 28,8 (D.P. $=15,2$ ) anos. Sendo assim, esses pacientes apresentaram em média 28,1 (D.P.=24,8) anos-maço de tabagismo.

A cirurgia realizada com maior frequência foi a RVM ( $n=60 ; 48 \%)$, seguida por troca de válvula ( $n=48$; $38,4 \%$ ), RVM combinada com troca de válvula ( $n=9 ; 7,2 \%$ ), troca combinada com plastia de válvula ( $n=4$; $3,2 \%$ ), plastia de válvula ( $n=3 ; 2,4 \%$ ) e RVM combinada com plastia de válvula ( $n=1 ; 0,8 \%$ ).

Na Tabela 3, apresentamos a distribuição dos pacientes, segundo as variáveis utilizadas para calcular o Sistema de Escore Clínico de Risco de Tuman. 
Tabela 3: Distribuição das variáveis utilizadas para calcular o Sistema de Escore Clínico de Risco de Tuman. Ribeirão Preto, SP, Brasil, agosto 2013 - fevereiro 2015.

\begin{tabular}{lcc}
\hline \multicolumn{1}{c}{ Fator pré-operatório } & Escore & Frequência $(\mathbf{n = 1 2 5}) \mathbf{n}(\%)$ \\
\hline $\begin{array}{l}\text { Cirurgia de emergência } \\
\text { Idade }\end{array}$ & 4 & zero \\
$\quad 65-74$ anos & 1 & $29(23,2)$ \\
$\quad \geq 75$ anos & 2 & $11(8,8)$ \\
Disfunção renal & 2 & $31(24,8)$ \\
Infarto do miocárdio prévio & & $12(9,6)$ \\
$\quad 3-6$ meses & 1 & $11(8,8)$ \\
$\quad<3$ meses & 2 & $43(34,4)$ \\
Sexo feminino & 2 & zero \\
Cirurgia cardíaca prévia & 2 & $06(4,8)$ \\
Hipertensão pulmonar & 2 & $03(2,4)$ \\
Doença cerebrovascular & 2 & $15(12,0)$ \\
Troca de válvula múltipla ou RVM ${ }^{a}+$ troca valvar & 2 & $61(48,8)$ \\
Troca de válvula aórtica ou mitral & $22(17,6)$ \\
Insuficiência cardíaca congestiva & 1 & $14(11,2)$ \\
Disfunção do ventrículo esquerdo & 1 & 2 \\
\hline
\end{tabular}

$\mathrm{RVM}^{\mathrm{a}}=$ Revascularização do Miocárdio

Com relação à classificação dos pacientes, segundo o risco de complicações e mortalidade após as cirurgias cardíacas, encontramos que 110 pacientes (88\%) apresentaram baixo risco (pontuação 0 - 5), cuja taxa de complicações esperada é de 14,6\% e taxa de mortalidade esperada de 3,3\%. O restante dos pacientes ( $n=15 ; 12 \%)$ foram classificados na categoria de moderado risco (pontuação 6-9) para o desenvolvimento de complicações e mortalidade pós-operatória, cujas taxas esperadas são 34,4\% e 10,0\%, respectivamente.

\section{DISCUSSÃO}

Nesta pesquisa, a maioria dos pacientes submetidos pela primeira vez às cirurgias cardíacas eletivas de RVM, correção de valvulopatias e RVM combinada com correção de valvulopatias apresentou baixo risco para o desenvolvimento de complicações pós-operatórias e mortalidade.

No estudo de validação do escore de $\operatorname{Tuman}^{(10)}$, no qual foram avaliados prospectivamente 296 pacientes submetidos à cirurgia cardíaca eletiva com uso de circulação extracorpórea, do tipo RVM e/ou procedimento valvar (implante de prótese ou cirurgia conservadora), os pesquisadores classificaram $85,1 \%$ dos pacientes como baixo risco para complicações pós-operatórias; 15,5\% como risco moderado e, apenas 1,0\% como alto risco. Esses resultados se assemelham aos encontrados neste artigo.

Após a validação, esse escore foi implantado na avaliação pré-operatória dos pacientes adultos submetidos à cirurgia cardíaca no Instituto do Coração de São Paulo (InCor), em 154 pacientes. Desse total, $63 \%(n=97)$ dos pacientes foram classificados como baixo risco; $35,7 \%(n=55)$ como moderado risco e $1,3 \%$ $(n=2)$ como alto risco para complicações e mortalidade pós-operatória ${ }^{(10)}$. Esses resultados também corroboram com os obtidos no presente estudo, com a maioria dos pacientes na categoria de baixo risco. Entretanto, em nosso estudo houve menor frequência de pacientes com risco moderado, provavelmente, devido à inclusão somente de pacientes submetidos à primeira cirurgia cardíaca e ainda, cirurgia eletiva. 
Conforme abordado anteriormente, o número reduzido de artigos que utilizaram escores validados na literatura para a classificação dos pacientes, segundo o risco de complicações e mortalidade após cirurgias cardíacas, dificultou a comparação dos nossos resultados ${ }^{(7,10)}$. Outra dificuldade encontrada foi o fato de que alguns autores investigaram exclusivamente pacientes submetidos à $\mathrm{RVM}^{(8,16)}$ ou exclusivamente submetidos a cirurgias de correção de valvopatias ${ }^{(6)}$. Além disso, outros pesquisadores investigaram apenas a mortalidade $\mathrm{e}^{(5-6,8,16)}$.

Em estudo realizado com o objetivo de identificar fatores de risco que influenciavam a mortalidade hospitalar de pacientes submetidos à cirurgia de implante de prótese valvar mecânica, os autores encontraram que as características significativamente relacionadas à mortalidade (taxa de 3,88\%) foram fração de ejeção do ventrículo esquerdo inferior a 30\%, lesão de válvula mitral e realização de cirurgia cardíaca prévia. A creatinina sérica foi significativamente maior nos pacientes que faleceram do que nos sobreviventes. Além disso, a cirurgia de troca de válvula concomitante com RVM, bem como a reoperação de urgência no pós-operatório, também foram correlacionadas significativamente com a mortalidade hospitalar ${ }^{(6)}$.

Com relação aos artigos que investigaram apenas pacientes submetidos à RVM, encontramos um estudo no qual os autores identificaram associação estatisticamente significante dos fatores idade (acima de 70 anos), internação pelo Sistema Único de Saúde (SUS), reoperação, cirurgia não eletiva, dependência de diálise, diabetes mellitus, creatinina sérica pré-operatória maior que $1,9 \mathrm{mg} / \mathrm{dl}$, insuficiência cardíaca congestiva, choque cardiogênico, edema agudo de pulmão e hipertensão pulmonar, com a mortalidade pósoperatória $^{(16)}$.

Em outra pesquisa desenvolvida com o objetivo de investigar a relação de características sociodemográficas com a mortalidade em pacientes submetidos à RVM, os autores encontraram que o sexo feminino, a idade (acima de 70 anos), a hipertensão arterial sistêmica, a doença renal, a doença cerebrovascular, as cirurgias de emergência, a realização de circulação extracorpórea e a reinternação na Unidade de Terapia Intensiva apresentaram efeito significativo sobre a mortalidade ${ }^{(8)}$.

Já em estudo desenvolvido no interior de São Paulo, os pesquisadores avaliaram, retrospectivamente, 783 prontuários de pacientes submetidos a cirurgias de RVM e/ou correção de valvopatias, com o objetivo de determinar os fatores de risco para mortalidade pós-operatória de pacientes septuagenários ou mais idosos. Do total de pacientes avaliados, $25 \%$ ( $n=197)$ tinham idade igual ou superior a 70 anos. Os fatores de risco preditivos da mortalidade pós-operatória, em pacientes septuagenários, foram sexo feminino, presença de doença pulmonar obstrutiva crônica, fração de ejeção do ventrículo esquerdo menor que $35 \%$, operação não eletiva e tempo de circulação extracorpórea maior que 120 minutos $^{(5)}$.

Dessa forma, nos estudos supracitados, alguns fatores foram similares com o Sistema de Escore Clínico de Risco de Tuman para avaliação do risco de complicações e mortalidade, nomeadamente: cirurgia de emergência ${ }^{(5,8,16)}$; idade ${ }^{(5,8,16)}$; disfunção renal ${ }^{(6,8,16)}$; sexo feminino ${ }^{(5,8)}$; cirurgia cardíaca prévia $^{(6,16)}$; hipertensão pulmonar $\left.{ }^{(16)}\right)$; doença cerebrovascular ${ }^{(8)}$; cirurgias de RVM com troca valvar ${ }^{(6)}$; insuficiência 
cardíaca congestiva ${ }^{(16)}$ e disfunção do ventrículo esquerdo ${ }^{(5-6)}$. Os fatores infarto do miocárdio prévio e troca de válvula aórtica ou mitral, presentes no do escore de Tuman, não foram descritos nos estudos.

Apesar das semelhanças de alguns fatores do Escore de Tuman com fatores descritos em outros estudos $^{(5-6,8,16)}$, devido à ausência de um escore ou uma categoria de risco, não foi possível comparar as amostras de nosso estudo considerando o risco de complicação ou mortalidade.

Encontramos ainda um estudo que criou um escore de classificação de risco para mortalidade pósoperatória de cirurgia cardíaca, considerando o perfil clínico pré-operatório do paciente e variáveis do intraoperatório e pós-operatório imediato. Segundo esse escore, a idade entre 65 e 74 anos (1 ponto), a idade acima de 75 anos ( 2 pontos), o diâmetro do átrio esquerdo acima de $45 \mathrm{~mm}$ (1 ponto), a creatinina préoperatória acima de $2 \mathrm{mg} / \mathrm{dl}$ ( 2 pontos), o tempo de circulação extracorpórea acima de 180 minutos ( 2 pontos), a razão da pressão parcial de oxigênio com a fração inspirada de oxigênio menor que 100 (3 pontos), o uso de aminas ( 3 pontos) e o tempo de ventilação mecânica maior que 12 horas ( 1 ponto) são preditores de mortalidade pós-operatória. Segundo esse escore, pacientes que pontuam de $0-2$ apresentam baixo risco de mortalidade, cuja taxa esperada é de 3,5\%; pacientes que pontuam de $3-5$ apresentam médio risco de mortalidade, cuja taxa esperada é de $7,5 \%$ e, por fim, pacientes que pontuam 6 ou mais são considerados de alto risco, com a taxa de mortalidade esperada maior que $15 \%{ }^{(7)}$.

As principais limitações deste estudo estão relacionadas com o número reduzido de pacientes investigados, além da exclusão dos pacientes que aguardavam uma reoperação cardíaca de qualquer natureza e que foram submetidos às cirurgias cardíacas de urgência/emergência. A exclusão desses pacientes é justificada pelo fato de esse ser um primeiro estudo em que buscamos avaliar o risco de complicações e mortalidade de pacientes em menor risco (submetidos a primeira cirurgia cardíaca e eletiva) e, também, pelo interesse em avaliar pacientes submetidos às cirurgias mais frequentes no referido hospital.

O desenvolvimento de novos estudos, com abordagens longitudinais, para verificar o valor preditivo desse escore para os pacientes estudados, faz-se necessário. Além disso, é importante a inclusão de pacientes que aguardam reoperação cardíaca e que tiveram a indicação de urgência ou emergência para a realização da cirurgia. Essas inclusões poderão trazer resultados mais abrangentes da população estudada.

\section{CONCLUSÃO}

A maioria dos pacientes investigados neste estudo apresentou baixo risco de desenvolvimento de complicações e mortalidade após cirurgias cardíacas eletivas de RVM, correção de valvulopatias e RVM combinada com correção de valvulopatias. A taxa de complicações esperada para esses pacientes é de 14,6\%, ao passo que a taxa de mortalidade esperada é de $3,3 \%$.

Atualmente, um estudo longitudinal está sendo desenvolvido, com o objetivo de investigar as principais complicações que esses pacientes desenvolveram no pós-operatório, bem como está sendo investigado o número de óbitos para o cálculo da taxa de mortalidade pós-operatória. Os resultados do estudo em andamento poderão confirmar o valor preditivo do escore utilizado e classificado no presente 
estudo, validando, assim, uma importante ferramenta para auxílio na decisão terapêutica, no planejamento da assistência de enfermagem, e na adoção de estratégias individualizadas para a prevenção de complicações e mortalidade pós-operatória.

\section{FINANCIAMENTO}

Estudo subvencionado pela Fundação de Amparado à Pesquisa do Estado de São Paulo.

\section{REFERÊNCIAS}

1. Villela LCM, Gomes FE, Meléndez JGV. Mortality trend due to cardiovascular, ischemic heart diseases, and cerebrovascular disease. J Nurs UFPE [Internet]. 2014 [acesso em: 31 mar. 2016]; 8(9):3134-41. Disponível em: http://www.revista.ufpe.br/revistaenfermagem/index.php/revista/article/viewArticle/4949.

2. DATASUS [Internet]. Brasília: Ministério da Saúde; 2016 [acesso em: 31 mar. 2016]. Disponível em:

http://www.datasus.gov.br.

3. Soares GMT, Ferreira DCS, Gonçalves MPC, Alves TGS, David FL, Henriques KMC et al. Prevalência das principais complicações pós-operatórias em cirurgias cardíacas. Rev Bras Cardiol [Internet]. 2011 [acesso em: 31 mar.

2016];24(3):139-46. Disponível em:

http://sociedades.cardiol.br/socerj/revista/2011 03/a_2011_v24 n03 01prevalencia.pdf.

4. Nishimura RA, Otto CM, Bonow RO, Carabello BA, Erwin JP, Guyton RA, et al. 2014 AHA/ACC Guideline for the Management of Patients With Valvular Heart Disease: Executive Summary: A Report of the American College of Cardiology/American Heart Association Task Force on Practice Guidelines. Circulation [Internet]. 2014 [acesso em: 31 mar. 2016];129(23):2440-92. Disponível em: http://dx.doi.org/10.1161/CIR.00000000000000029.

5. Alves Júnior L, Rodrigues AJ, Évora PRB, Basseto S, Scorzoni Filho A, Luciano PM, et al.Risk factors in septuagenarians or elderly patients undergone coronary artery bypass grafting and or valves operations. Rev Bras Cir Cardiovasc. [Internet]. 2008 [acesso em: 31 mar. 2016];23(4):550-5. Disponível em: http://dx.doi.org/10.1590/s010276382008000400016 .

6. Bacco MW, Sartori AP, Sant'Anna JRM, Santos MF, Prates PR, Kalil RAK, et al.Risk factors for hospital mortality in valve replacement with mechanical prosthesis. Rev Bras Cir Cardiovasc. [Internet]. 2009 [acesso em: 31 mar. 2016];24(3):334-40. Disponível em: http://dx.doi.org/10.1590/\$0102-76382009000400012.

7. Gomes RV, Tura B, Mendonça Filho TF, Campos LAA, Rouge A, Nogueira PMM et al. Rioescore: escore preditivo de mortalidade para pacientes submetidos à cirurgia cardíaca baseado em variáveis de pré, per e primeiro dia de pósoperatório. Rev. SOCERJ [Internet]. 2005 [acesso em: 31 mar. 2016];18(6):516-26. Disponível em:

http://www.rbconline.org.br/artigo/rioescore-escore-preditivo-de-mortalidade-para-pacientes-submetidos-a-cirurgiacardiaca-baseado-em-variaveis-de-pre-per-e-primeiro-dia-de-pos-operatorio/.

8. Oliveira EL, Westphal GA, Mastroeni MF. Demographic and clinical characteristics of patients undergoing coronary artery bypass graft surgery and their relation to mortality. Rev Bras Cir Cardiovasc [Internet]. 2012 [acesso em: 31 mar. 2016];27(1):52-60. Disponível em: http://dx.doi.org/10.5935/1678-9741.20120009.

9. Piegas LS, Bittar OJNV, Haddad N. Myocardial revascularization surgery (MRS). Results from national health system (SUS). Arq. Bras. Cardiol. [Internet]. 2009 [acesso em: 31 mar. 2016];93(5):555-60. Disponível em:

http://dx.doi.org/10.1590/s0066-782X2009001100018.

10. Strabelli TMV, Stolf NAG, Uip DE. Uso prático de um índice de risco de complicações após cirurgia cardíaca. Arq. Bras. Cardiol. [Internet]. 2008 [acesso em: 31 mar. 2016];91(5):342-7. Disponível em:

http://dx.doi.org/10.1590/S0066-782X2008001700010.

11. Secretaria de Estado Saúde, Governo do Estado de São Paulo [Internet]. São Paulo: Governo do Estado de São Paulo (BR) [acesso em: 31 mar. 2016]. DRS XIII - Ribeirão Preto. Disponível em:

http://www.saude.sp.gov.br/ses/institucional/departamentos-regionais-de-saude/drs-xiii-ribeirao-preto.

12. Folstein MF, Folstein SE, McHugh PR. "Mini-mental state". A practical method for grading the cognitive state of patients for the clinician. J Psychiatr Res [Internet]. 1975 [acesso em: 31 mar. 2016];12(3):189-98. Disponível em: http://dx.doi.org/10.1016/0022-3956(75)90026-6. 
13. Brucki SMD, Nitrini R, Caramelli P, Bertolucci PHF, Okamoto IH. Sugestões para o uso do mini-exame do estado mental no Brasil. Arq. Neuro-Psiquiatr. [Internet]. 2003 [acesso em: 31 mar. 2016];61(3B):777-81. Disponível em: http://dx.doi.org/10.1590/S0004-282X2003000500014.

14. Peto, R. Influence of dose and duration of smoke in lung cancer rates. In: Zaridze D, Peto R. Tobacco: a major international health hazard. Lyon (France): International Agency for Research on Cancer; 1986.

15. Tuman KJ, Mccarthy RJ, March RJ, Najafi H, Ivanikovich A.D. Morbidity and duration of ICU stay after cardiac surgery. A model for preoperative risk assessment. Chest [Internet]. 1992 [acesso em: 31 mar. 2016];102(1):36-44. Disponível em: http://dx.doi.org/10.1378/chest.102.1.36.

16. Almeida FF, Barreto SM, Couto BRGM, Starling CEF. Predictive factors of in-hospital mortality and of severe perioperative complications in myocardial revascularization surgery. Arq. Bras. Cardiol. [Internet]. 2003 [acesso em: 31 mar. 2016];80(1):51-60. Disponível em: http://dx.doi.org/10.1590/S0066-782X2003000100005. 